\title{
Special issue on convergence of real-time 3D imaging and optical communications
}

\author{
Sanghyun Seo $\cdot$ Changhoon Lee $\cdot$ Xilin Chen
}

Published online: 12 June 2014

(C) Springer-Verlag Berlin Heidelberg 2014

With the fast developments in electronics industry and the amazing growing of the community of potential customers, multimedia are increasingly ubiquitous: an increasing number of people are faced with a life in a world accompanied by Internet pop-ups and streaming television, mobile phone texting and video clips and pod-casting. Rapid advancements in computer graphics, imaging, multimedia service and game technologies determine the influence and impact of digital images on modern society tremendously, and image processing and computer graphics are now a key component in science and technology. Image processing has become such an essential component in contemporary science and technology that many tasks would not be conceivable without it. The rapid progress in handling digital images by computer or human, and the associated developments in related analysis methods, has propelled computer vision into one of the most important sub-fields in scientific imaging. It is a truly interdisciplinary subject that draws from synergistic developments involving many disciplines and is used in computer graphics, computer vision, computer art and many other fields.

\footnotetext{
S. Seo $(\bowtie)$

Electronics and Telecommunications Research Institute (ETRI), Daejeon, Korea

e-mail: shseo75@gmail.com

C. Lee

Seoul National University of Science and Technology,

Seoul, Republic of Korea

e-mail: changhoonlee08@gmail.com

X. Chen

Chinese Academy of Sciences, Beijing, China

e-mail: xlchen@ict.ac.cn
}

This special issue is intended to foster the dissemination of state-of-the-art research in the area of "Convergence of Real-Time 3D Imaging and Optical Communications" that can fulfill the anytime and anywhere access expectations of the consumers. Original research articles are solicited in all aspects of Real-Time 3D Imaging and Optical Communication technologies including emerging trends and applications, theoretical studies, and experimental prototypes. This special issue consists of seven papers addressed in this guest editorial by brief summaries.

The first paper entitled "Analyzing Repetitive Action in Game based on Sequence Pattern Matching" by S. Kim et al. presents a log-based usability evaluation system to analyze user behavior in a gaming environment. They explore the potential of input log data for automated usability evaluation and visualization of player behavior in a game. They traced the keyboard input value and mouse movement of users involving a sequence data-mining technique in a gaming environment. Additionally, they constructed 3D body meshes for behavior analysis using Kinect interface and visualized the data obtained by tracing and automatic searching of repetitive patterns in the game for further analysis. Especially, this can be applied to bot detection and player identification in online gaming because the approach proposes measuring the distribution of frequent patterns by the mutual information between the distributions being observed for certain players.

The second paper entitled in this issue "Development of a Virtual Aquarium System Interacting with a Smart Device" by Y. Seo et al. proposes an interactive virtual aquarium system that interacts with a smart device as a control interface. They developed a virtual aquarium graphic system having a remote interaction application of a smart device and performed an experiment that demonstrates the feasibility and the effectiveness of the proposed 
system as an example of a new type of interactive application of a smart display. The proposed system provides a blueprint for the future environment. It is also expected that the various features and contents that connect with the $\mathrm{u}$-home, u-healthcare, and service robots in a u-environment will be available on smart devices soon.

The third paper entitled "Real-time Emotion Retrieval Scheme in Video with Image Sequence Features" by J. Chang et al. introduces a new real-time emotion retrieval scheme in video making use of image sequence features consisting of color information, key frame extraction, video sound, and optical flow. These feature values are combined with a weight function to decide the emotion of the video stream. Even though the result of the experiments does not always represent the correct emotion of the video because personal emotion related to the same video content is very subjective.

The fourth paper entitled "Efficient Paper Mosaic Rendering on Mobile devices Based on Position-based tiling" by M. Han et al. proposes an accelerated method that generates tiles for colored paper mosaic application on mobile devices. The proposed method focuses on processing in real-time by locating tiles taking into consideration the edges of the image and the shapes of neighbor tiles. The proposed algorithm demonstrates the generation process and proposes an ordering method similar to human processing.

The fifth paper entitled "OUB: A Scalable Grid-Based Surface-representation for Real-time High-resolution Rendering" by J. Choi et al. presents a scalable solution for highresolution surface representation of a 3D surface based on GPU for real-time application. To achieve this, they introduce an Orthogonal Unified Buffer (OUB), which is an extension of the Layered Depth Cube (LDC) as an alternative to the Orthogonal Frame Buffer (OFB). The proposed OUB system achieves significant improvement in scalability, which enables further high resolution compared to the OFB. It also guarantees plausible memory efficiency, independent of an object's shape or topology. This algorithm is well suited for real-time applications: high-resolution 3-D painting, curvature estimation and particle system based on the OUB mentioning just some characteristics.

The sixth paper entitled "Real-time Video Photomosaics with Optimized Image Set and GPU" by Y. Choi et al. introduces a real-time video photo-mosaics method using a genetic feature selection method based on an optimized image set and CUDA GPU acceleration. The system proposes three approaches to increase the image generation frame rate: pattern search based on CUDA, assembling an optimized image subset based on genetic feature selection method and consistency preservation for reducing the computational cost.

The seventh and final paper entitled "Quality Analysis of Real-Time Digital Broadcasting Images Applying the QoE
Measurement Technology" by B. Hong et al. analyzes the effect of the evaluation cycle on the "mean opinion score (MOS)", and proposes a method of selecting an appropriate evaluation cycle for the subjective video quality evaluation of digital broadcasting (IPTV, DCATV, satellite broadcasting). This paper also measures the real-time video quality of local commercial broadcasting services by applying various evaluation types.

Finally, our special thanks go to both Chief Editors of the Journals of Real-Time Image Processing, Nasser Kehtarnavaz and Matthias F. Carlsohn, and all editorial staff for their valuable support throughout the preparation and publication of this special issue. We would like to thank all authors for their contributions to this special issue and to the external reviewers for their excellent help in reviewing the manuscripts. We hope that the selected papers will contribute to the on-going research in convergence of realtime imaging in academia, industry, and other interested agencies and organizations.

Sanghyun Seo received his B.S. degrees in Computer Science and Engineering from ChungAng University, Seoul, Korea, in 1998 and M.S. and Ph.D. degrees in GSAIM at ChungAng University, Seoul, Korea, in 2000 and 2010. He was senior researcher at G-Inno System from 2002 to 2005 . He was the postdoctoral researcher at ChungAng University, in 2010 and the postdoctoral researcher at LIRIS Lab, Lyon 1 University from Feb. 2011 to Feb 2013. Now, he is working at the ETRI (Electronics and Telecommunications Research Institute), DaeJeon, Korea. His research interests are in the area of computer graphics, non-photorealistic rendering and animation, 3D GIS system, real-time rendering using GPU and game technology. He has been a reviewer in Multimedia Tools and Applications (MTAP), Computer and Graphics UK (Elsevier), Journal of Supercomputing (JOS), Visual Computer (Springer), and Program member in many international Conferences and workshops.

Changhoon Lee received his Ph.D. degree in Graduate School of Information Management and Security (GSIMS) from Korea University, Korea. He is now an assistant professor in Department of Computer Science and Engineering, Seoul National University of Science and Technology, Korea. Prior to this, he was a research professor in Center for information Security and Technology, Korea University, Korea in 2008. From 2009 till 2010, he was a full-time lecturer in the School of Computer Engineering of Hanshin University, Korea and was an assistant professor in the School of Computer Engineering of Hanshin University, Korea in 2011. He has also served as chairs and program committees for many international conferences and workshops. He has been serving as a guest editor for international journals by some publishers. His research interests include information security, cryptography, digital forensics, convergence security, etc.

Xilin Chen received the BS, MS, and Ph.D. degrees in Computer Science from Harbin Institute of Technology, China, in 1988, 1991, and 1994, respectively. Then he joined the Department of Computer Science and Engineering, Harbin Institute of Technology, where he was a lecturer (1994), associate professor (1996) and professor (1999-2005). He was a visiting scholar with Carnegie Mellon University from 2001 to 2004 . He was elected into the 100 Talents Program of Chinese Academy of Sciences (CAS). He is now the 
director of the Key Laboratory of Intelligent Information Processing, CAS. His research interests are Image Understanding, Computer Vision, Pattern Recognition, Image Processing, Multimodal Interface, and Digital Video Broadcasting. And he received several awards, including thrice of the China's State Scientific and Technological
Progress Award in 2000, 2003 and 2005, respectively, for his academic researches. He has served as a program committee member for more than 40 international conferences, and is the (co)author of more than 200 papers. He is a senior member of the IEEE. 